\title{
ANÁLISIS DIAGNÓSTICO DE LAS DOTACIONES DE BOMBEROS EN LA COMARCA DE L'ALACANTÍ: PROPUESTAS
}

\author{
Carles Baeza Llaneras \\ Facultad de Filosofía y Letras. Universidad de Alicante \\ Baezacarles4@gmail.com
}

\section{RESUMEN}

La comarca de l'Alacantí queda inserta en una zona en la que los riesgos naturales se manifiestan con asiduidad. En las últimas décadas se ha vivido un gran avance en cuanto a la prevención del riesgo se refiere, debido al aumento de legislación relacionada. Las organizaciones de bomberos representan una parte imprescindible de la gestión de emergencias al ser uno de los actores que intervienen directamente cuando aparece alguna. Por este motivo la situación de las infraestructuras desde donde operan tiene un valor determinante a la hora de pasar a la acción. Estableciendo una estimación del tiempo de respuesta aproximado de cada parque de bomberos en la comarca de l'Alacantí, se puede vislumbrar qué zonas reciben una menor cobertura. Este primer acercamiento permite advertir que dichas zonas requieren de un análisis más en detalle para mejorar su situación.

Palabras Clave: Riesgos naturales; parque de bomberos; tiempo de respuesta; propuesta.

\section{ABSTRACT}

\section{Diagnostic analysis of fire stations in the Alacantí district: proposals}

The Alacantí region is an area where natural risks are common. During recent decades great progress has been made in risk prevention thanks to improvements in environmental law. Firefighters are an essential part of hazard management because they are the first to attend when any emergency arises. For this reason, the location of fire stations is of great importance in relation to their response time. Studying and improving the estimated response time of each station of the Alacantí region will be helpful in order to improve the monitoring of areas with less coverage. This first approach notes which areas need a more specific analysis to improve their situation.

Key words: Natural hazards; fire station; response time; proposal.

\section{INTRODUCCIÓN}

La comarca de l'Alacantí o Campo de Alicante contiene tanto municipios costeros, como son El Campello y el propio Alicante, municipios que se encuentran en una zona a caballo entre el interior y la costa, como son Sant Joan d'Alacant, Mutxamel y Sant Vicent del Raspeig y municipios situados al interior como Aigües de Busot, Busot, Agost, la Torre de les Maçanes y Xixona. Este territorio comarcal se sitúa al Sur-Este de la provincia de Alicante, ubicación que expone a esta comarca a ciertos procesos físicos peligrosos característicos de las tierras del mediterráneo (Olcina Cantos, 2008). La ocupación en las últimas décadas de zonas en donde se desarrollan este tipo de procesos desemboca en un aumento de la vulnerabilidad y por tanto del riesgo, aumentando la necesidad de un sistema de gestión de emergencias eficiente.

Por lo general, los ejemplos con mayor repercusión ocurridos hasta la fecha en la comarca de l'Alacantí se caracterizan por tener un origen atmosférico, lo que no quiere decir que este territorio no quede expuesto a riesgos de diferente tipología. Algunos de los sucesos más desastrosos que han ocurrido en la comarca son, por ejemplo, el del 27 de septiembre de 1966 cuando el Observatorio de Ciudad Jardín rec- 
ogió una intensidad de precipitación máxima de $240 \mathrm{~mm}$ en 24 horas. Este episodio propició que varias calles de la ciudad de Alicante se transformaran en el lugar por donde discurrían notorios volúmenes de agua. Por otro lado el que se desarrolló entre los días 19 y 20 de Octubre de 1982, que afectó a la ciudad de Alicante de una manera particularmente destacable ya que se descargaron un total de $220 \mathrm{~mm}$ el día 20, según el mismo observatorio (Olcina Cantos, 1994). Cabe destacar que el ayuntamiento de Alicante estimó pérdidas de 1.200 millones de pesetas de las propiedades municipales, sin tener en cuenta los costes de reparación de las infraestructuras que pertenecían al estado.

Figura 1. Mapa de localización de la comarca de l'Alacantí.

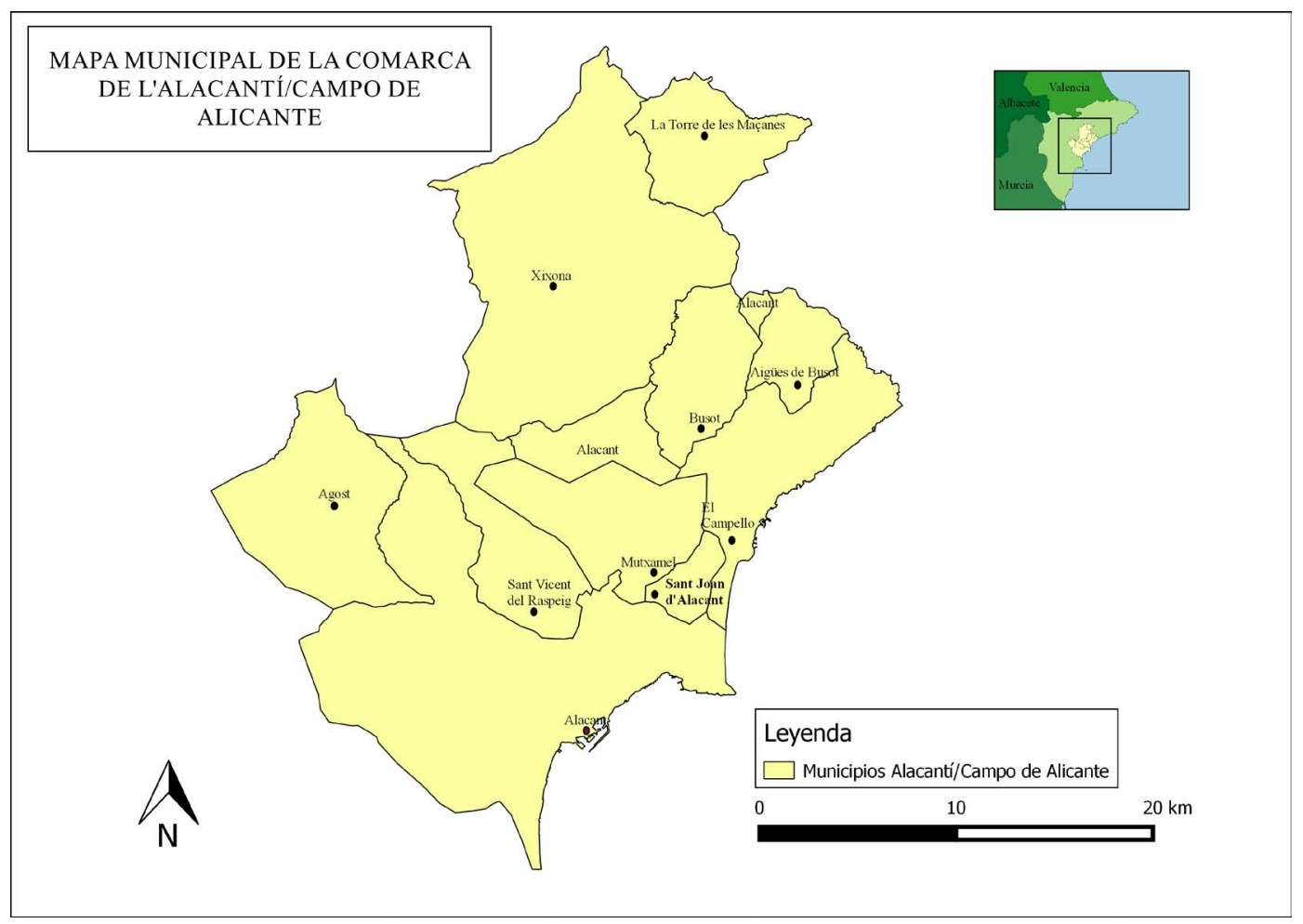

Elaboración propia.

El análisis de las dotaciones responde a un primer acercamiento de la situación de las mismas. Se vislumbrará, según la metodología expuesta por el autor qué zonas podrían estar en una situación de falta de cobertura. El fin de esta primera toma de contacto será justificar la existencia de futuros análisis con un nivel de detalle mayor, que ayuden a mejorar la posible falta de cobertura del servicio de bomberos. Finalmente, con la información obtenida será posible la recomendación de zonas que podrían ser óptimas para establecer nuevas infraestructuras de bomberos, llevándose (como en el caso anterior) un examen más minucioso de sus características.

\section{METODOLOGÍA}

El auge de la tecnología ha supuesto el desarrollo de la cartografía digital. Las herramientas de este tipo permiten que el desarrollo de los análisis espaciales se aborden con mapas quizás más complejos y detallados, realizados en un tiempo mucho menor que los convencionales. Un análisis exhaustivo de isócronas ha de incorporar varios factores, como puede ser todo aquello que gira entorno a las vías (dirección, tipo velocidad oficial, zonas de concentración de accidentes...etc.).

De la misma forma ocurre para situar parques de bomberos, estaciones de policía u hospitales, debido a la estrecha relación que tienen estos centros con la rapidez de actuación de sus operarios en ciertas situaciones. Como exponen Kanoun, Chabchoub y Aouni (2010) la efectividad de un sistema de emergencias suele depender del tamaño de la flota de la que se disponga, la ubicación de los centros y la estrategia previa. Aunque no se encuentra directamente relacionado con el establecimiento de isócronas, un estudio 
a considerar es el llevado a cabo por Rodríguez Núñez y Gutiérrez Puebla (2012). En él se presentan una serie de escenarios hipotéticos en donde se ven interrumpidas ciertas vías, pretendiéndose analizar cuáles pueden ser sus consecuencias. Para ello las variables concebidas son los tipos de vía y la densidad de población, utilizando la función OD Matrix Cost y siendo plasmado en cartografía mediante el ArcGis 9.3.

De forma similar al análisis anterior, Bono y Gutiérrez (2011) analizan el estado de la accesibilidad de las carreteras después del terremoto de Haití en las zonas de Príncipe y Carrefour. Es interesante concebir que según el escenario a analizar las variables pueden cambiar de forma sustancial aunque la naturaleza del estudio sea la misma. En este caso, no se han tenido en cuenta la dirección de las carreteras ya que en el área de estudio predominan las unidireccionales, además de obviar que el ahorro temporal prima sobre las leyes viales.

Otro ejemplo de modelo en el que se tienen en cuenta las vías es el desarrollado por Özdmar, Ekinci y Küçükyazici (2004) con el que se pretende planificar el reparto eficiente de los materiales necesarios al desarrollarse un riesgo natural en un área regional. En el modelo se tiene en cuenta el tiempo, dependiendo de la demanda de las materias primas según la situación, y se integra una red de flujo lineal para los materiales y otra entera para los vehículos. Se aplica a una situación real, el terremoto de Izmit en el año 1999.

Además de tener como finalidad el estudio de las vías, los modelos matemáticos y los Sistemas de Información Geográfica [SIG] -en inglés GIS- han sido utilizados para establecer un criterio óptimo a la hora de situar infraestructuras. En este caso, los edificios a tener en cuenta son las dotaciones de bomberos, mereciendo especial atención debido a la importancia de optimizar el tiempo de respuesta. Un precursor en este tipo de estudios es el llevado a cabo por Schreuder (1981), en la ciudad de Rotterdam. Significó un avance sustancial en la planificación de los parques de bomberos, teniendo como objetivo las situaciones de los mismos así como de autobombas.

Otro ejemplo concreto y actual es el estudio llevado a cabo por Erden y Coskun (2010), que combina el método AHP y los SIG. Se tiene en cuenta la densidad de población, la proximidad a vías principales, la distancia a los parques de bomberos existentes, instalaciones con materiales peligrosos, densidad de edificios construidos con madera y las áreas sujetas a peligros por terremoto.

Hay que tener en cuenta que el objetivo de este trabajo es un primer acercamiento al análisis de la situación de los parques de bomberos actuales en la comarca de l'Alacantí, así como al futuro emplazamiento de nuevas instalaciones. Por este motivo el método utilizado aborda el tema de una manera superficial.

El protocolo de extinción de incendios en l'Alacantí se divide entre el Servicio de Prevención y Extinción de Incendios y Salvamento [SPEIS] y el Consorcio Provincial de Bomberos. Cada organización concibe su propia área de actuación, siendo la ciudad de Alacant en el caso del SPEIS, y el resto de municipios de la comarca de l'Alacantí en el caso del Consorcio Provincial. Si se observa un mapa de la situación de los parques de bomberos en la comarca alicantina se puede llegar a la prematura conclusión de que existe una polarización de los mismos. Por este motivo es necesario fundamentar esta afirmación considerando el tiempo de respuesta de cada parque.

Con la aplicación "Google Maps" y su herramienta "Direcciones" se ha extraído el tiempo que tarda un vehículo desde cada uno de los parques de bomberos, hasta una serie de puntos concretos. Una vez sabido esto, se han digitalizado dichos puntos adjudicándoles un valor según la información recogida.

De esta manera a partir del programa QuantumGis se ha utilizado la función IDW (Distancia Inversa Ponderada) para, a partir de estos puntos, realizar una interpolación que estime el tiempo para las zonas cercanas a cada uno. Se han extraído alrededor de 500 puntos para cada figura. Cabe mencionar que la información utilizada por la aplicación para calcular los trayectos, no tiene un carácter público.

Sin embargo, Russel (2013), quien trabajó para la empresa Google desde el año 2007 a 2010, describió de forma general que información se utiliza. Algunos de los factores mencionados son la velocidad oficial de las vías, las velocidades recomendadas, datos históricos de velocidades en ciertos periodos de tiempo o "información del tráfico a tiempo real". También destaca que se "mezclan los datos de cualquier fuente disponible para llegar a la mejor predicción". En cualquier caso, la utilización de esta herramienta, combinada con un sistema de información geográfica [SIG] permite extraer de forma sencilla una primera impresión del tiempo de respuesta de los lugares deseados, y concretar que zonas merecen ser objeto de un estudio con mayor detalle. 


\section{RESULTADOS}

Cabe destacar que el lugar donde se ha situado el nuevo Parque Central de Central del SPEIS es óptimo para minimizar el tiempo de respuesta en la zona litoral y prelitoral norte de la ciudad de Alacant. Esta zona, que alcanza su máxima población durante la época estival, se caracteriza principalmente por la situación de diseminados de viviendas unifamiliares. Sin embargo, el número de los bloques de edificios de viviendas también es elevado. En suma, esta zona constituye un gran núcleo de población durante cualquier época del año, aumentando de manera significativa con la entrada del turismo durante el verano. Por tanto, es importante que el tiempo de respuesta frente a una emergencia sea relativamente bajo. No obstante, la situación del nuevo parque en la zona del Cabo de las Huertas ha podido polarizar las isócronas de respuesta, aumentando el tiempo de las mismas hacia la zona sur de la ciudad.

Figura 2. Zonas afectadas en la ciudad de Alacant

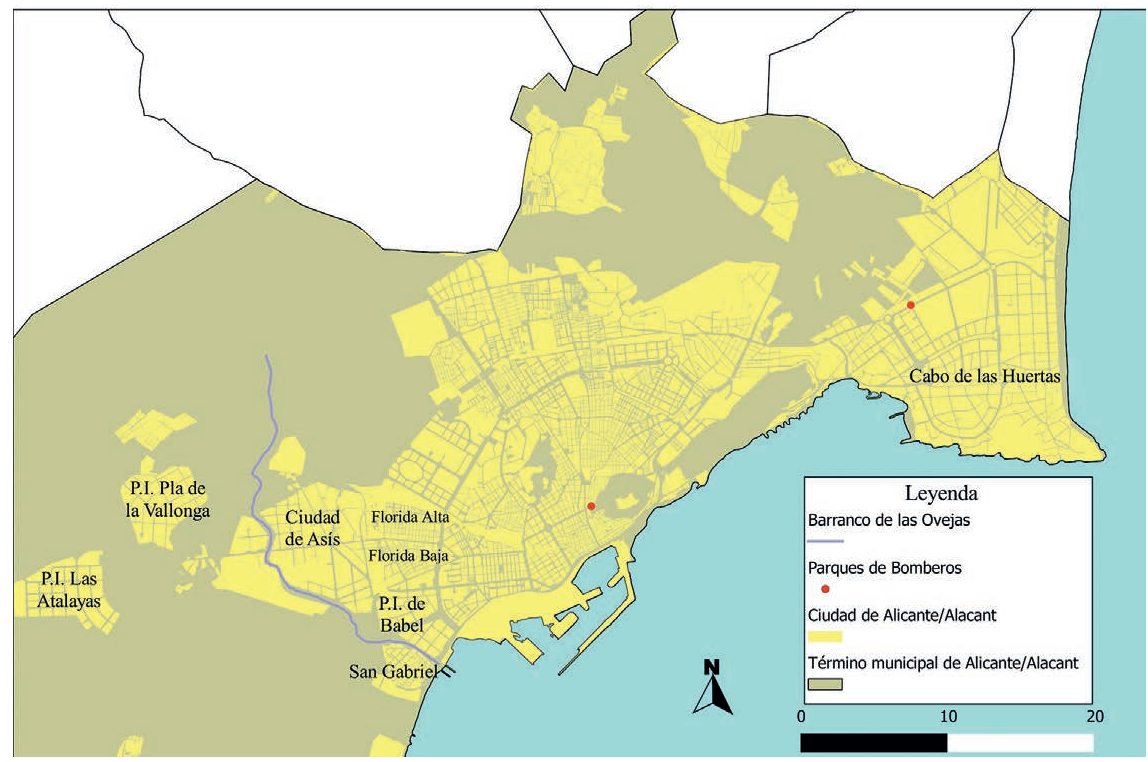

Elaboración propia.

Figura 3. Cobertura del nuevo parque de bomberos del SPEIS

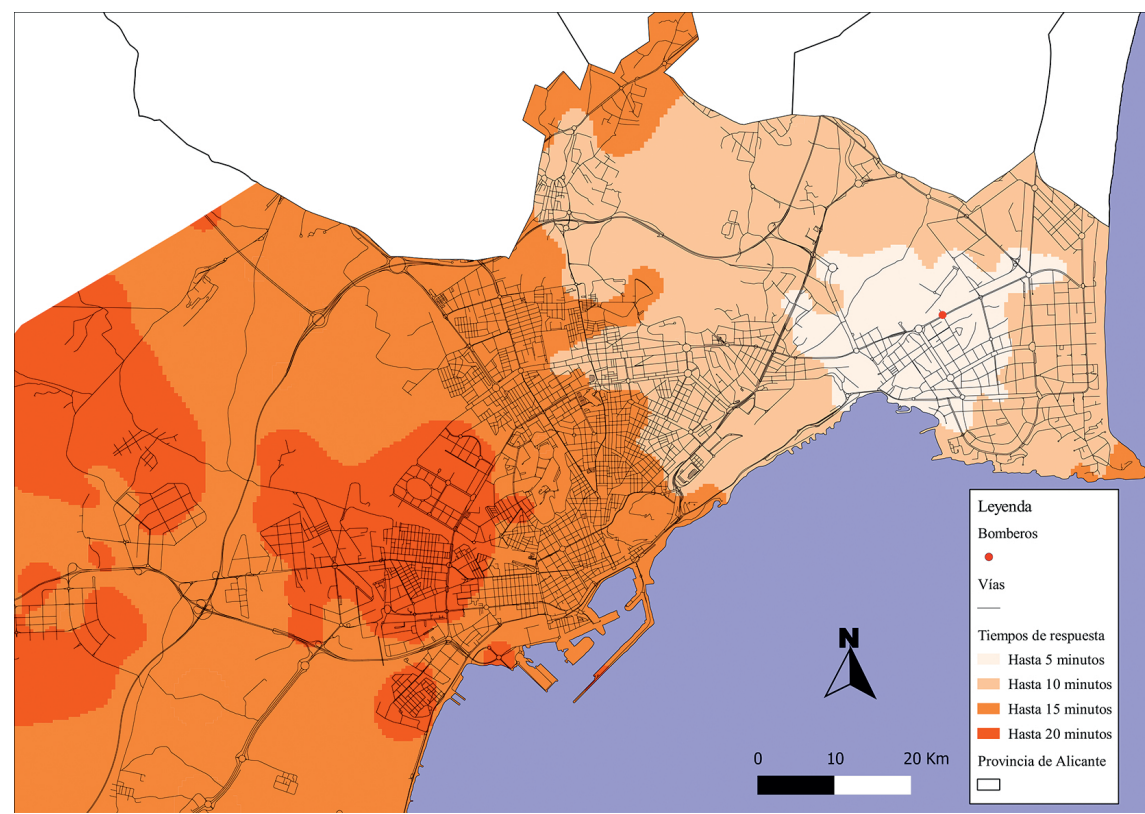

Elaboración propia. 
En la Figura 2 se representan los lugares que han visto como su cobertura ha variado después de la situación del nuevo parque. Así en la Figura 3, se observa que la mitad noreste de la ciudad de Alacant, queda inmersa en una zona con un tiempo de respuesta de hasta 10 minutos. Además incluida dentro de esta, existe una subzona cuyo tiempo de respuesta es de hasta 5 minutos. Lógicamente este lugar corresponde con la situación del nuevo parque, que como se puede comprobar ha mejorado sustancialmente la cobertura de mitad noroeste de la ciudad, y más concretamente la del distrito del Cabo de las Huertas. En la mitad suroeste el tiempo de respuesta comienza a aumentar, dejando sumergida a gran parte de la ciudad en un área de hasta 15 minutos, ejemplo de ello son los barrios de Florida Alta y Florida Baja. Por otro lado, el sur de la ciudad se encuentra con una cobertura quizás insuficiente, concretamente los polígonos del Pla de la Vallonga, Babel y las Atalayas, así como el barrio de Ciudad de Asís, que puede responder hasta un máximo de 20 minutos.

Es cierto que existe otro parque operativo en la ciudad de Alacant (el cual se a continuación) que consigue reducir el tiempo de respuesta hacia la zona más central de la ciudad. Pese a ello, es de gran utilidad concebir el tiempo de respuesta de las infraestructuras por separado, debido a que esto permite conocer el máximo potencial de las mismas y en conclusión descubrir que zonas sufren en ambos casos una menor cobertura. Además, es interesante saber cómo podría ser la respuesta de los parques individualmente, al poder producirse una hipotética situación de inoperatividad o de desbordamiento de recursos de uno de los mismos.

En la Figura 4, se denota la situación del parque cercano al centro de la ciudad de Alacant. Dicho núcleo, queda rodeado de una zona con una cobertura de hasta 10 minutos, donde se incluye otra cuyo tiempo de respuesta es de hasta 5 minutos. El aumento ocurre, de forma parecida al caso interior, en las zonas exteriores la ciudad. En este caso tanto los barrios de Florida Alta y Florida Baja y el polígono industrial de Babel se engloban dentro de un tiempo con un máximo de hasta 15 minutos, que aunque siendo inferior al caso anterior, continua significando un hecho a tener en cuenta. En el caso de los polígonos industriales de Las Atalayas y el Pla de la Vallonga, así como el barrio de ciudad de Asís, el tiempo estimado sigue siendo de hasta 20 minutos. Es necesario mencionar que en el Cabo de las Huertas, se describe una situación de hasta 20 minutos de demora, en un territorio que excede ligeramente la primera línea de costa.

Figura 4. Cobertura del parque de bomberos de la zona central de Alicante

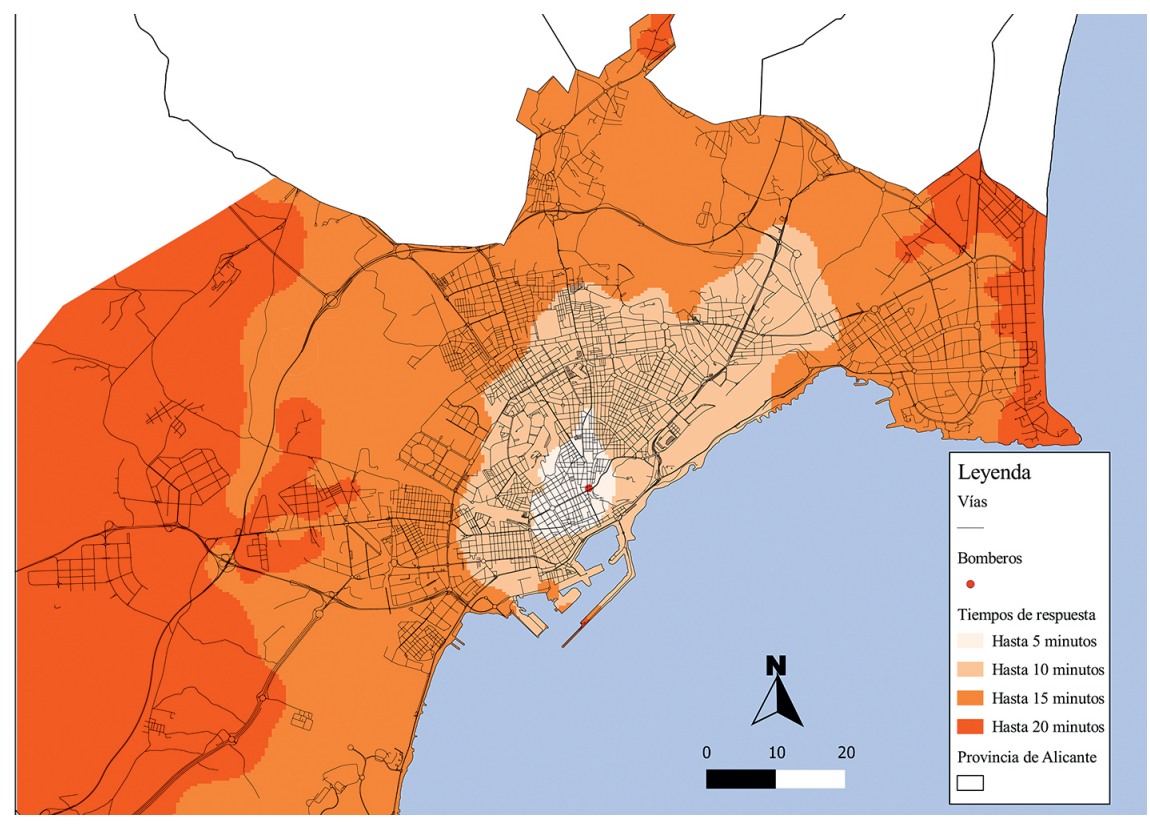

Elaboración propia.

De la misma forma que en el ejemplo anterior, se han estimado las isocronas de respuesta para un caso que atañe al Consorcio Provincial de Bomberos de Alicante, que actúa para el resto de la comarca. Dicho caso corresponde con los núcleos poblacionales del municipio de El Campello.

La cobertura de esta zona es en general insuficiente, debido a que la menor demora que existe en el municipio es de 16 minutos. A partir de esta aclaración, se puede decir que en general, el municipio queda inmerso en una zona cuyo tiempo de respuesta es de 18 minutos. 
Además, cabe concretar que hacia el norte del mismo, donde se dibuja un núcleo de urbanizaciones, el tiempo de respuesta excede los 20 minutos un hecho realmente destacable y que no puede pasar desapercibido.

Figura 5. Cobertura del municipio alicantino de El Campello

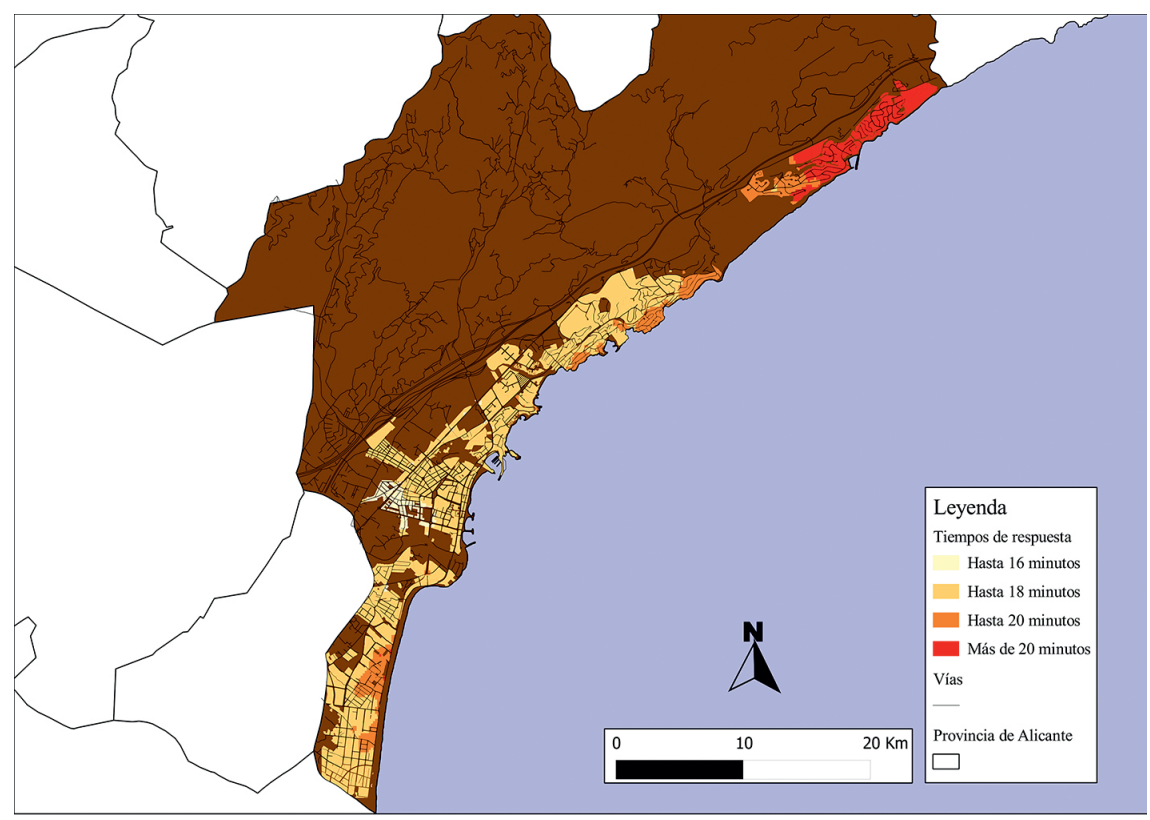

Elaboración propia.

\section{DISCUSIÓN DE RESULTADOS}

El territorio de la comarca de l>Alacantí está incluido en una zona con riesgos naturales diversos y latentes. En cuanto a la situación de los parques de los servicios de bomberos, se ha de tener en cuenta qué zonas quedan más alejadas de los mismos y qué ideas podrían tomarse en consideración para tratar de reducir el tiempo de respuesta. En primer lugar, como se ha podido comprobar el nuevo Parque Central del SPEIS ofrece cobertura a una zona que ha adquirido población y ha desarrollado de forma considerable su entramado urbano en las últimas dos décadas: El Cabo de las Huertas.

Por otra parte, la situación de dicha infraestructura en este lugar ha podido conllevar que las zonas situadas al sur de la ciudad, que albergan varios polígonos industriales, puedan quedar relativamente aisladas al producirse una emergencia. De forma parecida ocurre con el Parque del Consorcio de Bomberos de Alicante en Sant Vicent del Raspeig. Teniendo en cuenta el ejemplo de El Campello, se puede reflexionar acerca de la situación del resto de los municipios de la comarca, muchos de los cuales se encuentran en zonas del interior de la montaña alicantina y cuya accesibilidad puede resultar dificultosa.

Como apunta Ochoa Monzó (1995) tanto la ordenación territorial como urbanística son medidas preventivas de riesgo y por tanto desempeñan un trabajo crucial a la hora de combatir las emergencias. Por este motivo, cabe tener en cuenta las zonas más comprometidas, convirtiéndolas en objeto de análisis para la situación de nuevas infraestructuras de gestión de la emergencia. Utilizando la información extraída del estudio, y a pesar de que escoger un lugar para una dotación de tales características requiere de un análisis mucho más detallado, se pueden estimar qué lugares podrían ser merecedores de futuros estudios para la situación de nuevas infraestructuras.

\section{CONCLUSIONES}

En primer lugar se ha escogido el pueblo de Xixona (Zona 1) debido esencialmente a su localización. Inmersa en una zona que corresponde con 12 minutos en el tiempo de respuesta, significa a priori una falta de cobertura destacable para una zona del interior de la comarca.

Por este motivo sería conveniente llevar cabo un análisis para corroborar si la colocación de un pequeño retén de bomberos en este municipio sería adecuado para mejorar. La situación céntrica de este 
municipio podría motivar la colocación del retén, creando un área de influencia equitativa para el interior de l'Alacantí. En segundo lugar, el municipio elegido ha sido El Campello (Zona 2).

El gran motivo que induce a situar otra pequeña infraestructura es la existencia de diseminados unifamiliares, sobretodo al norte del municipio. El ejemplo más claro es el de la "Coveta Fumà", urbanización costera que constituye un lugar relativamente alejado de los servicios de bomberos, con una caótica distribución de las calles y el parcelario, lo que agrava su situación. Así, la situación de un retén de bomberos podría establecerse en el núcleo urbano de El Campello, viéndose ampliamente potenciado si el Consorcio Provincial y el SPEIS, funcionaran como la misma organización, tema que se mencionará más adelante. Además de ofrecer cobertura sobre estos "puntos calientes", los municipios de Aigües y Busot complementarían su cobertura, sirviendo de nexo entre las zonas limítrofes a la costa y el interior.

En tercer lugar la ciudad de Alacant (Zona 3) debería de incluir en su parte sur otro pequeño retén. Como se ha ido comentando, dicha zona contiene varios polígonos industriales, lo que aumenta notablemente el riesgo, en caso de desarrollarse una emergencia. La existencia del Barranco de las Ovejas agrava el estado de la cuestión, al ser posible su activación mediante fenómenos de precipitación torrencial. Este hecho puede suponer la inhabilitación de vías, lo que supone un aislamiento mayor de estos lugares. La falta de infraestructuras en este área, la buena conexión viaria de los polígonos y tal vez la reconversión de alguna de las naves podrían suponer un decrecimiento claro del tiempo de respuesta.

Figura 6. Propuesta de ubicación de nuevas infraestructuras.

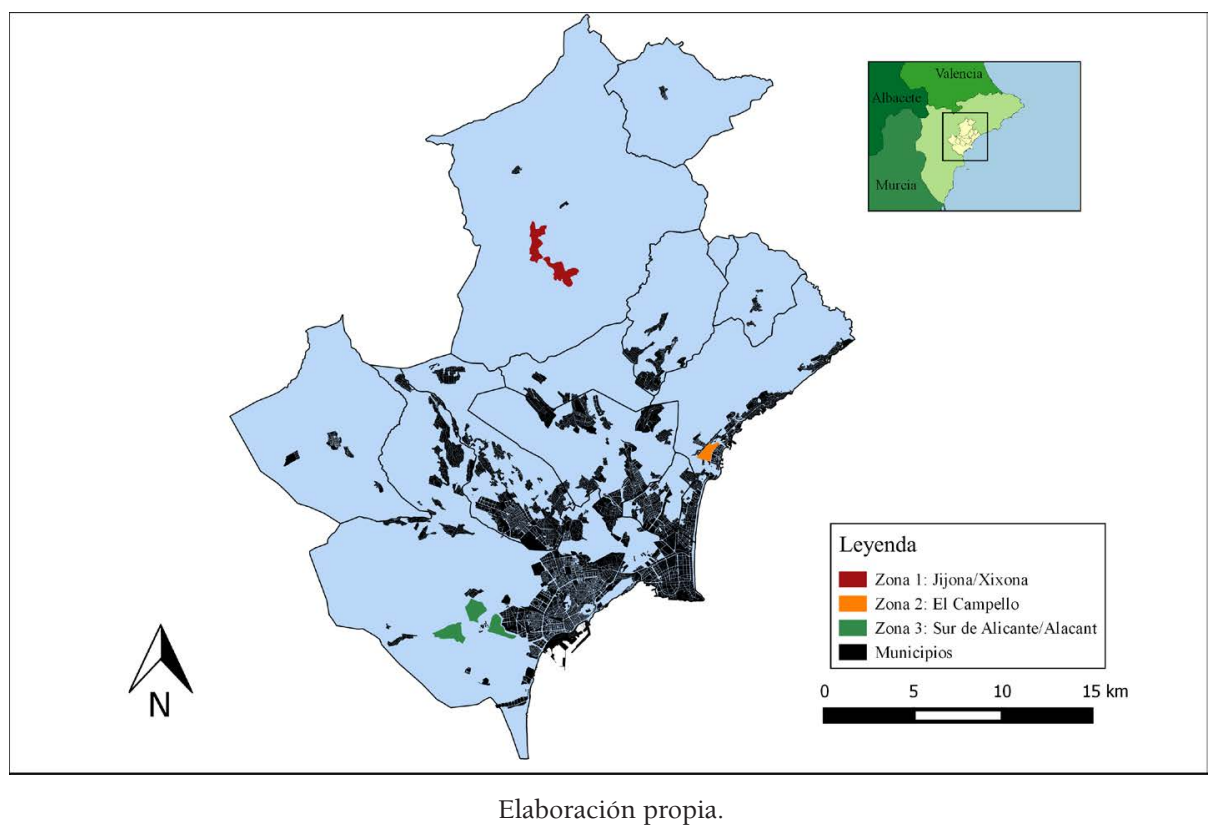

Cabe mencionar que esta situación produjo descontento entre la población del sur de la ciudad. Varias agrupaciones vecinales manifestaron su preocupación en relación con la polarización de los servicios de bomberos en la zona norte Alacant, incluso llevando el asunto al pleno municipal (Lahoz, 2014). Finalmente, se puede señalar una idea de carácter estructural que sería adecuado contemplar sería la de unir las dos organizaciones de bomberos que actúan en la comarca. En definitiva, se trata de mejorar las relaciones entre administraciones y la gobernanza en una cuestión fundamental como es la gestión del riesgo y de las emergencias. La unión de ambas corporaciones supondría la creación de una red más amplia, que conllevaría una reducción del tiempo de respuesta.

\section{REFERENCIAS}

Bono, F. \& Gutiérrez, E. (2011). A network-based analysis of the impact of structural damage on urban accessibility following a disaster: the case of the seismically damaged Port Au Prince and Carrefour urban road networks. Journal of Transport Geography, 19, 1443-1455. Doi: http://dx.doi.org/10.1016/j. jtrangeo.2011.08.002 
Erden, T. \& Coskun, M.Z. (2010). Multi-criteria site selection for fire services: the interaction with analytic hierarchy process and geographic information systems. Natural Hazards and Earth System Sciences, 10, 2127-2134. Recuperado de http://www.nat-hazards-earth-syst-sci.net/10/2127/2010/ nhess-10-2127-2010.pdf

Kanoun, I., Chabchoub, H. \& Aouni, B. (2010). Goal programming model for fire emergency service facilities site selection. Infor, 48, 143-153. Recuperado de https://pdfs.semanticscholar.org/ c5e3/4949bdf06efa2022965ea20fad202a909335.pdf

Lahoz, E. M. (19 de noviembre de 2014). Vecinos de la zona sur y CC OO se unen para reclamar el subparque de bomberos. La Verdad. Recuperado de http://www.laverdad.es/alicante/ciudad-alicante/201411/19/ vecinos-zona-unen-para-20141119014115-v.html

Ochoa Monzó, J. (1995). El régimen Jurídico de los Riesgos Mayores. La protección Civil. (Tesis Doctoral). Recuperada de: http://hdl.handle.net/10045/3778

Olcina Cantos, J. (2008). El Mediterráneo, región-riesgo: una visión desde España. España y el Mediterráneo, una reflexión desde la geografía española: aportación española al XXXI Congreso de la Unión Geográfica Internacional, 29-34. Recuperado de http://hdl.handle.net/10045/23014

Olcina Cantos J. (1994). Tormentas y granizadas en las tierras alicantinas. Alicante: Publicaciones de la Universidad de Alicante

Özdmar, L., Ekinci E. \& Küçükyazici, B. (2004). Emergency logistics planning in natural disasters. Annals of Operations Research, 129. 217-245. http://dx.doi.org/10.1023/B:ANOR.0000030690.27939.39

Rodríguez Núñez, E. y Gutiérrez Puebla, J. (2012). Análisis de vulnerabilidad de redes de carreteras mediante indicadores de accesibilidad y SIG. Intensidad y polarización de los efectos del cierre de tramos en la red de carreteras de Mallorca. GeoFocus, 12, 374-394. Recuperado de http://geofocus. rediris.es/2012/Articulo15_2012.pdf

Russel, R. (2013). How does Google maps calculate your eta?. Quora. Recuperado de https://www.quora. com/How-does-Google-Maps-calculate-your-ETA

Schreuder, J. (1981). Application of a location model to fire stations in Rotterdam. European Journal of Operational Research, 6, 212-219. http://dx.doi.org/ 10.1007/978-3-642-73778-7_76 